\title{
Creencias de GÉNERo, PRÁCticas de CRIANZA y APOYO SOCIAL PERCIBIDO: EL CASO DE UNA PAREJA HOMOSEXUAL Y UNA HETEROSEXUAL
}

\section{Gender Beliefs, Parenting Practices and Perceived Social Support: The Case of a Homosexual and a Heterosexual Couple}

\author{
Juan Sebastian Rueda-Toro ${ }^{12}$, María Isabel Arana ${ }^{2}$, \\ Nathalia Buitrago ${ }^{2}$, María del Mar Sánchez ${ }^{2}$, \\ Ana María Pineda ${ }^{2}$, Linda Teresa Orcasita ${ }^{3}$
}

Recibido: 2019-09-26

Resumen: Este reporte de caso analizó las creencias de género, las prácticas de crianza y el apoyo social percibido por una pareja homosexual y una heterosexual de Cali-Colombia, mediante una investigación cualitativa con diseńo fenomenológico utilizando entrevistas semiestructuradas. A partir del análisis temático de los datos, se encontró que los hombres fueron relacionados con autoridad, responsabilidad, ser guía, carácter fuerte y ser estricto; las mujeres con ser ejemplo, apoyo, sabiduría, comprensión y afecto. Estas
Aceptado: 2019-12-13 creencias de género se mantenían en la idea del hombre fuerte-protector y la mujer afectiva-comprensiva. En las prácticas de crianza ambas parejas dialogaban y buscaban un acompańamiento afectuoso. Las creencias de género y las prácticas de crianza fueron similares en ambas parejas y se identificó la importancia del apoyo social familiar.

Palabras clave: creencias de género, prácticas de crianza, homoparentalidad, parentalidad, Cali-Colombia.
Para citar este artículo en APA: Rueda-Toro, J., Arana, M., Buitrago, N., Sánchez, M., Pineda, A. y

Orcasita, L. (2019). Creencias de género, prácticas de crianza y apoyo social percibido: el caso de una pareja homosexual y una heterosexual.

Revista de Psicología Universidad de Antioquia, 11(2), 151-176. doi: 10.17533/udea.rp.v11n2a06
Joven investigador Minciencias (convocatoria 850 de 2019) línea Familias, Género y Sexualidad del Grupo de Investigación Bienestar, Trabajo, Cultura y Sociedad (BITACUS). Correo: sebastianrueda@javerianacali.edu.co; https://orcid. org/0000-0003-3824-4310

2 Estudiantes de Psicología, Pontificia Universidad Javeriana Cali, Colombia.

3 Psicóloga y Magister en Familia de la Pontificia Universidad Javeriana Cali, Colombia. Directora de la Línea Familias, Género y Sexualidad del Grupo de Investigación Bienestar, Trabajo, Cultura y Sociedad (BITACUS). Correo: ltorcasita@javerianacali.edu.co; https://orcid.org/0000-0002-75999280 


\begin{abstract}
This case report analyzed the gender beliefs, parenting practices and perceived social support of a homosexual and a heterosexual couple from Cali-Colombia, through a qualitative research with phenomenological design using semi-structured interviews. From the thematic analysis of the data, it was found that men were related to authority, responsibility, being a guide, having a strong character and being strict; women were with being an example, support, wisdom, understanding and affection. These beliefs remai-
\end{abstract}

ned in the idea of the strong-protective man and the affective-understanding woman. In parenting practices, both couples said they look for giving their children affectionate accompaniment. Gender beliefs and parenting practices were similar in both couples and it was identified the importance of family social support.

Keywords: gender beliefs, parenting practices, homoparentality, parenthood, Cali-Colombia.

\section{Introducción}

De acuerdo con el Colegio Colombiano de Psicólogos [Colpsic] (2014), las familias homoparentales y sus dinámicas de interacción han sido investigadas principalmente en países como Estados Unidos, Reino Unido y Bélgica. El reconocimiento de las familias homoparentales es un tema reciente en Colombia, por lo cual son escasos los estudios acerca de los efectos que tiene la crianza por parte de parejas del mismo sexo en el desarrollo integral de niños, niñas y adolescentes. Es por esto que este artículo busca acercarse a las particularidades de la crianza de los hijos en familias diversas, y contribuir a estudios posteriores en el proceso de generación y aplicación de lineamientos de políticas públicas a partir de estrategias que permitan combatir las inequidades de género y discriminación por diversidad sexual y de géneros en el contexto colombiano (González, Posada, Rojas, Rueda y Soto, 2017).

A nivel internacional el estudio de familias homoparentales y sus dinámicas tiene un importante recorrido. Por ejemplo, en España una de las preocupaciones e inquietudes principales que comparten los padres y madres homosexuales en torno al bienestar de sus hijos e hijas, radica en las posibles consecuencias negativas y experiencias de estigma que su orientación sexual pueda acarrear a los menores. Sin embargo, las familias homoparentales se sienten capacitadas para educar y criar satisfactoriamente a sus hijos e hijas, dejando claro que su orientación sexual no es un indicador que sirva para evaluar su crianza (Ceballos, 2012). 
En este mismo país, Smietana (2010) buscó analizar las cinco dimensiones de los actos comunicativos que ocurrieron entre trece familias homoparentales y las comunidades escolares de sus hijos. Cada familia asumió una de tres estrategias de comunicación: abierta al hablar del tema de la orientación sexual (OS) con cualquier persona, selectiva al escoger a las personas a quienes se comunica o no y no comunicación al no hablar sobre la OS. La comunicación abierta tenía efectos positivos en la relación entre los padres y sus hijos, mientras que, respecto a los efectos de las estrategias restantes, si bien ayudaron a que los hijos no sufrieran de bullying de sus compañeros de clase debido a la orientación sexual de sus padres/madres, al no saber que pertenecían a una familia homoparental, se registró que dentro del contexto familiar se experimentaban limitaciones y tensiones en las relaciones; además, las redes de apoyo de estas familias eran limitadas en comparación con las familias que preferían comunicarse abiertamente.

Por otro lado, en un estudio realizado en la Universidad Nacional Autónoma de México (UNAM) por Esquila, Zarza, Villafańa y Oudhof (2015), se encontró que el hombre suele ser considerado como proveedor de recursos del hogar y autoridad principal, mientras que la mujer es vista como la responsable de la casa y de los hijos. Además, existen criterios de feminidad y masculinidad que buscan cumplir mujeres y hombres, respectivamente. En el caso de la mujer se asocia a lo subjetivo, intuitivo, pasivo, tierno, sensible, dócil, receptivo, empático, dependiente, emocional y conservador; mientras que el hombre es visto como el encargado de tomar las decisiones familiares, la aportación de los ingresos económicos y la capacidad de resolver asuntos del ámbito público. Así, se hace relevante el estudio del género en relación con la crianza de los hijos, especialmente en familias homoparentales, para distinguir la influencia que pueden llegar a tener las creencias de género de los padres y madres sobre los hijos e hijas, a través de las prácticas de crianza que estos utilizan.

Laguna (2018) llevó a cabo una investigación en Ciudad de México para conocer la manera en que hombres homosexuales acceden a la crianza y a arreglos parentales frente a limitaciones biológicas, sociales y legales. En sus hallazgos, el autor resalta que los hombres gais emplean formas novedosas de 
ejercer su parentalidad, mientras afrontan problemas relacionados con la homofobia, el estigma y los prejuicios frente a la diversidad sexo-genérica. Estas nuevas formas de parentalidad implicaban: la desgenerización del cuidado, es decir, asumir prácticas de cuidado sin importar que estas tradicionalmente fueran asignadas a las mujeres y no a los hombres; la desalienación de los roles tradicionales, consistente en la distribución de las tareas de cuidado de los hijos e hijas de acuerdo a condiciones particulares de la pareja como la vinculación laboral y el tiempo libre, por fuera de los roles de género tradicionales; y el apoyo por parte de redes no familiares, ya que la mayoría se desvinculan de sus familias de origen por el rechazo frente a su orientación sexual.

Acosta, Costales y Rosales (2017) muestran la presencia de legitimidad legal de parejas homosexuales que desean conformar una familia a partir de la adopción de un niño o niña, en Cuba; sin embargo, a nivel social aún existen múltiples detractores. Para los autores, si bien las parejas homosexuales tienen derecho a conformar una familia, Latinoamérica continúa siendo un territorio que invalida socialmente tal derecho porque posee una larga tradición homofóbica y machista. Finalmente, afirman que la orientación sexual no debería ser un criterio para establecer que una pareja puede o no criar a un niño o niña, pues este constructo hace referencia únicamente a las preferencias afectivas y eróticas de la persona y no se relaciona necesariamente con las capacidades que pueda tener para criar a sus hijos e hijas.

Ríos (2017) realizó un estudio con siete familias homoparentales en Perú y encontró que la motivación para la conformación de pareja iba más allá de la satisfacción sexual, ya que implicaba asumir responsabilidades mutuas. Además, para estas parejas la familia no involucraba solamente un factor biológico de descendencia, sino que se constituía a través de un compromiso voluntario. En relación con las formas de crianza, las madres lesbianas presentaban un discurso basado en la protección de los niños frente a un entorno que los podía llegar a dañar y se posicionaban como expertas en la crianza, mientras que los padres gais preferían enfrentar los problemas manteniendo una posición constante de aprendizaje frente a los retos emergentes de ser padres. Esto se relaciona con los planteamientos de Herrera, Miranda, Pavicevic y Sciaraffia (2018), quienes al explorar las experiencias de paternidad de 14 hombres gais 
en Chile encontraron que estas no difieren significativamente de las de padres heterosexuales; solo eran distintas porque los padres gais debían enfrentarse a estereotipos culturales que imponen que es necesaria la presencia de una mujer para la crianza de un niño o niña y a tener que proteger a sus familias de los prejuicios y estigmas existentes en un entorno que consideran hostil.

En el contexto colombiano la mayoría de las investigaciones han sido de carácter teórico, lo que pone de manifiesto la importancia de generar estudios empíricos que permitan un acercamiento a las subjetividades de arreglos familiares distintos a los heteroparentales (Andrade y Uribe, 2015). Gómez (2004) afirma la importancia de hablar de modelos familiares en lugar de un único tipo de familia, debido a los cambios y transformaciones que el último siglo ha conllevado en esta institución. De esta manera, existen diversas tipologías de hogares que pueden ser clasificadas de acuerdo con la estructura familiar; han surgido arreglos biparentales de familias distintos al núcleo tradicional conformado por una pareja heterosexual y sus hijos, como lo son las parejas homoparentales.

El observatorio de Políticas de las Familias (OPF) en un informe titulado Tipologías de Familias en Colombia: Evolución 1993 - 2014, señaló que en 2014 los hogares biparentales continuaban siendo los más representativos de la población colombiana con un 46,3\% del total, de los cuales el 0,12\% eran parejas del mismo sexo con hijos e hijas (OPF y Departamento Nacional de Planeación [DNP], 2015). Esto constituye tanto una realidad emergente como un reto, pues, como lo plantean Andrade y Uribe (2015) en un artículo teórico acerca del "cuidado de las familias homoparentales como una forma de responder a las presiones heteronormativas del contexto occidental que descalifica y rechaza las diversas constituciones familiares" (p. 1), tradicionalmente se ha definido a las familias de una manera que implica importantes limitaciones para su comprensión e intervención desde la diversidad, especialmente en occidente, en donde la constitución de este grupo humano ha sido asumida desde la heterosexualidad, dejando a un lado configuraciones familiares como las homoparentales.

Zapata (2013) exploró las percepciones de las condiciones de crianza de padres homosexuales en Colombia y encontró que la crianza homoparental 
se relacionaba con "el ejercicio de enseñar, dar ejemplo, orientar, sancionar, observar los comportamientos de los hijos y las hijas, preocuparse, intercambiar expresiones afectivas con ellas y ellos" (p. 11). Asimismo, encontró relatos asociados con el miedo y la esperanza, además de resaltar el papel que juega la sociedad: "los niños, las niñas y los jóvenes que crecen en familias homoparentales enfrentan una difícil situación” (p. 15) debido a que las prácticas de crianza de parejas del mismo sexo aún son estigmatizadas y, en muchas ocasiones, discriminadas.

Acevedo-Correa et al. (2018) buscaron abordar la adopción homoparental desde la competencia de los padres para la crianza, teniendo en cuenta la normativa jurídica actual alrededor de este tema en Colombia, y, con base en la existencia del derecho de las personas homosexuales a conformar una familia, se reconoció al componente sexual como algo separado de la capacidad de crianza.

Martínez (2018), en una revisión de la literatura científica entre 1990 y 2017 que buscaba establecer una comparación entre el ajuste psicológico, el desarrollo de la sexualidad, la estigmatización y el desempeńo cognitivo entre niños biológicos y adoptados por parejas del mismo sexo, identifica una tendencia de los estudios a no reconocer diferencias en estos grupos de familias, a excepción del grado de estigmatización que es una variable dependiente de factores sociales externos al núcleo familiar. Castaño-Suárez, Sánchez-Trujillo y Viveros-Chavarría (2018), en una revisión que buscaba analizar la literatura científica latinoamericana entorno a las prácticas de crianza de familias homoparentales, concluyeron que la paternidad y maternidad en dichas parejas presentan similitudes a las de parejas heterosexuales, lo que nuevamente separa la preferencia sexual de estas personas de sus capacidades de crianza. Los autores argumentan que las parejas del mismo sexo son funcionales en la crianza de sus hijos e hijas y que el desconocimiento de otras formas de convivir en familias distintas a la nuclear constituye un problema social aún vigente en Colombia.

En esta dirección, resulta relevante destacar el concepto de familia, que para Jelin (1998) es una institución social que regula, canaliza y otorga significado social y cultural a necesidades como la sexualidad, la procreación y la 
convivencia; aspectos que han generado una creciente heterogeneidad social. Para algunas personas, la familia tradicional es la única forma posible de familia y pensar en otras conformaciones puede significar una problemática. Sin embargo, gracias a las transformaciones sociales que han tenido lugar durante el último siglo, se evidencia la importancia de hablar de modelos familiares, más que de un único modelo familiar; como lo plantea Gómez (2004), se puede hablar de familias monoparentales, familias reconstituidas y familias multiétnicas, así como de la concepción de hijos mediante vías alternativas como la adopción o la reproducción asistida.

Anudado a ello se menciona que la orientación sexual es un constructo psicológico que explica la atracción erótica, afectiva y emocional hacia las personas del sexo contrario, del mismo sexo o de ambos sexos (Córdoba, 2019). Por tanto, Gómez (2004) expone que dentro de las familias modernas se deben incluir las familias lesbigay, término adoptado del inglés que sirve para definir a las familias cuyos progenitores son gais, bisexuales o lesbianas, y cuyos hijos crecen y se desarrollan en su seno.

Es dentro del contexto de las familias donde se aprenden las diferencias de roles atribuidos a hombres y mujeres, las actitudes hacia los sexos y los comportamientos sexuales aceptados o rechazados por la sociedad. Esto se da a través de la transmisión de pautas de socialización radicadas en la familia por medio de la comunicación, dimensión fundamental de la vida del ser humano y proceso familiar que se encarga de tejer puentes que favorecen escenarios de construcción de relaciones entre las personas. Por lo anterior, el género, las prácticas de crianza y el apoyo social percibido son dimensiones vinculadas a la construcción relacional mediante las dinámicas familiares que se gestan en los procesos de convivencia.

De acuerdo con García (2007) el género como categoría social se ha organizado y estructurado a partir de la diferenciación de las masculinidades y feminidades vinculadas desde las características corporales: se pasa de lo biológico a lo sociocultural, de ahí que aparezcan expectativas de comportamiento y actuación diferenciadas entre ambos sexos. Por su parte, las creencias de género son entendidas como productos subjetivos del pensamiento frente a los que se tiene un firme asentimiento y conformidad, en relación con "los atributos, las 
actividades, las conductas y los roles establecidos socialmente que una sociedad en particular considera apropiados para niños y hombres, o niñas y mujeres los cuales influyen en la manera en que las personas actúan, interactúan y en cómo se sienten sobre sí mismas" (APA, 2017 p. 1).

En la propuesta de Izzedin y Pachajoa (2009) las prácticas de crianza son portadoras de significaciones sociales, se relacionan con la normatividad y la forma de educar que siguen los padres frente al comportamiento de los hijos. Estas prácticas de crianza son caracterizadas en tres componentes: la comunicación, el cuidado y la educación.

La comunicación tiene un papel muy importante en la formación y la funcionalidad de la familia, pues por medio de ésta se dan los diálogos en los que se transmite a los niños los límites y los roles que se tienen dentro de la familia, (Garcés y Palacio, 2010), asimismo, constituye una de las formas más conocidas de expresar el cariño, además de los actos físicos. Con respecto al cuidado, de acuerdo con el Instituto Colombiano de Bienestar Familiar (ICBF) (2006): “(...) se trata de un acompañamiento afectuoso e inteligente por parte de los padres y los adultos significativos, fundamentado en el cariño, la tradición cultural, el sentido común y algunos conocimientos científicos" (p. 07). Y la educación, según Izzedin y Pachajoa (2009), se relaciona con la normatividad y forma de educar que siguen los padres frente al comportamiento de los hijos, siendo portadoras de significaciones sociales.

Finalmente, el apoyo social percibido por ambas parejas en la crianza de los hijos hace referencia a la evaluación subjetiva y objetiva de la red de apoyo que tiene una persona, teniendo en cuenta su familia, amigos, instituciones, entre otros actores de la sociedad; es la dimensión subjetiva compuesta por la cognición y evaluación del apoyo en cuanto a la satisfacción y adecuación a sus necesidades (Orcasita y Uribe, 2010). Específicamente, se abordó el apoyo emocional, entendido como intimidad, apego, confort, cuidado y preocupación; y el apoyo informacional, definido como consejo, guía o información (Orcasita y Uribe, 2010).

Teniendo en cuenta los antecedentes y referentes teóricos revisados se hace necesaria una aproximación a la realidad del contexto latinoamericano y colombiano, y al estigma, prejuicio, discriminación y rechazo que aún se 
generan frente a familias con una estructura diferente a la nuclear. Diversos estudios afirman que aún persisten creencias conservadoras y hegemónicas que posicionan la homosexualidad como producto de arreglos familiares disfuncionales (Astaíza-Martínez, 2016), y que las representaciones sociales frente a los géneros aún prevalecen desde el sexo biológico y la tradición hegemónica de discriminación hacia lo femenino, rechazo hacia formas distintas de ser hombre y mujer en el mundo y permanencia de relaciones de poder entre los sexos, posicionando a las mujeres desde categorías como ser de otro y no ser sexual (Sánchez y Cárdenas, 2017).

Esta investigación buscó analizar las creencias de género, las prácticas de crianza y el apoyo social percibido en la crianza de una pareja homosexual y una pareja heterosexual, con el fin de: (1) describir las creencias de género de una pareja homosexual y una heterosexual, (2) caracterizar las prácticas de crianza empleadas por cada pareja e (3) identificar las percepciones de cada una de las parejas entorno al apoyo social percibido en la crianza de sus hijos e hijas.

\section{Método}

\section{Tipo y diseño de investigación}

La investigación fue de tipo cualitativa y tuvo un diseño de estudio de caso fenomenológico, el cual buscó abarcar la complejidad de un caso particular entre muchos otros, pretendiendo la comprensión profunda de ese caso específico (Stake, 2007). El estudio de caso fenomenológico resulta pertinente cuando el objetivo que se persigue es la comprensión de la perspectiva de los participantes acerca de ciertas experiencias o profundizar en sus opiniones y vivencias (Hernández, Fernández y Baptista, 2010; Mieles, Tonon y Alvarado, 2012), en especial cuando el tema de estudio ha sido poco explorado como es el caso de Colombia ${ }^{3}$.

\footnotetext{
3 Los autores agradecen a la profesora Myriam Román Muñoz del curso "Investigación en Psicología III" que acompañó el proyecto de investigación del cual se deriva este artículo.
} 
[160] Juan Sebastian Rueda-Toro, María Isabel Arana, Nathalia Buitrago, María del Mar Sánchez, Ana María Pineda, Linda Teresa Orcasita

\section{Participantes}

Los participantes del estudio fueron dos parejas entre los 20 y 27 años, residentes en la ciudad de Cali, seleccionadas mediante un muestreo de tipo no probabilístico intencional por bola de nieve (Martínez-Salgado, 2012): una pareja homosexual (dos hombres) y otra heterosexual, cada una con una hija menor de 8 años y ambas residentes de la ciudad de Cali-Colombia. Los criterios de inclusión fueron: (1) que tuvieran mínimo un hijo o una hija (2) que los participantes tuviesen como mínimo un año de convivencia en pareja para garantizar un tiempo de ejercer roles de crianza juntos.

\section{Instrumento}

Se diseñó una guía de entrevista semiestructurada (Bonilla y Rodríguez, 2013) en la que se plantearon las categorías de análisis deductivas (1) creencias de género (García, 2007), (2) prácticas de crianza (Izzedin y Pachajoa, 2009) y (3) apoyo social percibido (Orcasita y Uribe, 2010), provenientes de la revisión de la literatura, las cuales permitían abarcar el fenómeno a estudiar, ahondar en cuestiones que surgían a lo largo de las entrevistas y realizar aclaraciones a través de la retroalimentación. El instrumento fue diseñado a partir de una revisión de estudios con objetivos y metodologías similares y, posteriormente, se llevó a cabo una validación por jueces expertos, lo que permitió ajustar la guía final de la entrevista.

\section{Procedimiento}

El trabajo de campo y análisis de las entrevistas se realizó a través de cuatro fases: (1) se contactó a las parejas participantes por medio de informantes clave, teniendo en cuenta los criterios de inclusión; (2) se solicitó la firma del consentimiento informado y las entrevistas, que fueron audio grabadas, se realizaron en un consultorio privado con una duración entre los 45 a 60 minutos; (3) se realizaron las transcripciones de las entrevistas, en donde los nombres de los participantes fueron reemplazados por seudónimos elegidos por ellos mismos para mantener la confidencialidad de la información, luego, 
los datos fueron reorganizados de acuerdo con las categorías y subcategorías a partir del análisis temático de las narrativas que consistió en identificar, organizar y analizar en detalle la información, para reportar temas que emergieron de una cuidadosa lectura y relectura de las entrevistas (Mieles et al., 2012); (4) los resultados fueron escritos de acuerdo con las categorías y subcategorías de análisis presentando los temas emergentes en cada una de estas, posteriormente se construyó la discusión y conclusiones del estudio.

\section{Consideraciones éticas}

La investigación estuvo orientada por las consideraciones éticas definidas en la resolución No. 008430 del Ministerio de Salud de Colombia (1993) que dicta las normas científicas, técnicas y administrativas sobre la investigación en salud y por el Manual Deontológico y Bioético de Psicología (Colpsic, 2009) y la ley 1090 del 2006 (Congreso de la República de Colombia, 2006), las cuales regulan el accionar de los psicólogos en Colombia. Del mismo modo, se consideró como un principio básico la confidencialidad de los participantes, para lo cual se usó un seudónimo escogido por ellos mismos y se recurrió a la firma de un consentimiento informado.

\section{Resultados}

Se presentan los resultados obtenidos de acuerdo con el objetivo de la investigación que fue analizar las creencias de género, las prácticas de crianza y el apoyo social percibido de una pareja homosexual y una heterosexual, apoyado en fragmentos de la narrativa de los participantes. El análisis empleado fue de tipo temático, por lo que después de organizar la información transcrita de acuerdo con las categorías de análisis deductivas a partir de la lectura y relectura de las entrevistas emergieron temas que son presentados a continuación. También, se retoma una categoría y una subcategoría emergentes que provienen de las narrativas de las parejas. 
[162] Juan Sebastian Rueda-Toro, María Isabel Arana, Nathalia Buitrago, María del Mar Sánchez, Ana María Pineda, Linda Teresa Orcasita

\section{Creencias de género}

En las creencias de género asociadas al rol que desempeñan los hombres en la sociedad, ambas parejas mencionaron la característica de autoridad y frente al contexto económico fue representativo el concepto de responsabilidad:

(...) la cabeza del hogar, como la persona que va a ser el principal apoyo, tanto económicamente como sentimentalmente en cierta forma (...) es fuerte y rudo, (...) el hombre tiene el rol económico porque obviamente si es la cabeza del hogar tiene que responder por su hogar y tratar de sacar adelante a sus hijos y en realidad todo lo económico, porque los papás son los que le dan el estudio, lo que viste y lo que tiene (Rick, pareja homosexual).

Ambas parejas atribuyeron el carácter y el rol de guía a los hombres dentro del hogar. Ante esto, un participante señaló que lo importante en el hogar no es el sexo de los integrantes de la pareja, sino el cariño y la comprensión que se tengan entre ellos:

(...) el rol que tiene el hombre es el de criar en todo sentido a su familia, dar ejemplo siempre...siempre guiar a su familia tanto espiritual como fisicamente, en todos los sentidos tiene que dar ejemplo (Andrés, pareja heterosexual).

No importa que sea hombre o que sea mujer, lo importante es que lo quieran a uno y que le quieran los hijos, eso es lo importante, ¿de qué me serviría a mi tener mujer y tener una familia "normal" entre comillas, si no habia respeto, si no habia un cariño, sino habia comprensión, si no habia un diálogo? (Rick, pareja homosexual).

El papel del hombre en la crianza de los hijos fue entendido por ambas parejas como estricto, refiriéndose a este como quién debe mostrar un rol de fuerza y autoridad hacia los hijos e hijas:

Es la autoridad y por medio de esa autoridad, si es un papá que tiene buena autoridad, que se para duro con la niña, ella va a sentir de cierta manera seguridad (...). El papá es básicamente el que regaña (Emilia, pareja heterosexual).

(...) y yo a mi hija le he pegado, cuando de verdad se lo merece, no por cualquier cosa le voy a pegar, no; cuando de verdad hace algo que pasa los límites la corrijo, porque yo quiero criar una niña de bien, quiero criar una niña que tenga valores, que tenga moral ¿me entiendes? (Pepe, pareja homosexual). 
Creencias de género, prácticas de crianza y apoyo social percibido: el caso de una pareja homosexual y una heterosexual

Por otra parte, el rol de la mujer en la sociedad fue relacionado por ambas parejas con ser un ejemplo y una representante de que es posible lograr lo que se desea; mientras que en el contexto económico y del hogar se consideró a las mujeres como apoyo, sabiduría y comprensión:

(...) la mujer es como ese ejemplo de que "cuando se quiere se puede" y que no es lo que la gente diga y que no es como tiene que ser, no, es como yo quiero que sea y como mi corazón lo sienta (Pepe, pareja homosexual).

(...) en lo económico también, tiene que ser una lider, tiene que amar lo que hace $y$ asi obviamente lo económico va a explotar de la manera en que tiene que explotar (Andrés, pareja heterosexual).

En la crianza de los hijos los participantes se refirieron a la mujer como quien provee el afecto y se encarga de propiciar una buena comunicación entre los miembros de la familia:

(...) es como la reflexiva, la que comunica casi todo, la afectiva (Pepe, pareja homosexual).

A los hijos la mamá tiene que darles muchisimo amor y básicamente entenderlos al máximo, por eso dije lo de la parte de a veces ser el malo (Andrés, pareja heterosexual).

\section{Prácticas de crianza}

De acuerdo con Izzedin y Pachajoa (2009), esta categoría fue abordada mediante tres subcategorías: comunicación, cuidado y educación. La comunicación entre los padres homosexuales y el padre y madre sobre sus hijas fue percibida como buena, dado que decían utilizar el diálogo de manera constante:

\footnotetext{
(...) nos comunicamos mucho (...). Si le pasa algo a la niña y estoy yo, o si está él, nos comunicamos mucho. Lo que sabe Pepe o lo que la niña le cuenta a Pepe, él intenta contármelo a mí y estar los tres muy comunicados y muy unidos (Rick, pareja homosexual).

Bien, creo que nos comunicamos bien. Pues (...) digamos que en el caso de mi esposo y mío es como muy fácil porque trabajamos juntos todo el día, o sea todo el día estamos juntos, entonces como que tenemos fluidez en esa parte, todo el día estamos hablando (Emilia, pareja heterosexual).
} 
[164] Juan Sebastian Rueda-Toro, María Isabel Arana, Nathalia Buitrago, María del Mar Sánchez, Ana María Pineda, Linda Teresa Orcasita

Frente al cuidado, ambas parejas manifestaron mantener una relación basada en el amor y en la confianza con sus hijas:

(...) es una relación de confianza, de mucho amor, de sí..como de mucho amor, esa es la palabra (Emilia, pareja heterosexual).

Sí considero que tiene la confianza de los dos, aunque a veces creo que le tiene un poquitico más de miedo a Pepe porque él hace el papel del hombre (...) Pero igual ella nos cuenta todo (Rick, pareja homosexual).

En relación con la educación, se hizo referencia al diálogo entre los padres para tomar decisiones y al establecimiento de normas dentro de la familia como una condición esencial para ambas parejas, encontrándose elementos comunes en lo que intentan transmitir a sus hijas como hábitos saludables, respeto, orden y solución de conflictos a través del diálogo:

Las normas las establecimos entre Pepe y yo dialogando, sabiendo que en todo hogar tiene que haber unas reglas (Rick, pareja homosexual).

Primero le comenzamos quitando beneficios, y digamos, no, que me quiero comer un helado: pues no, ya no te voy a comprar el helado porque te equivocaste, hiciste algo en el celular o lo que sea. Cuando ya es demasiado, que ya está muy fuerte la rebeldía, yo la pongo en un asiento y no la dejo bajarse hasta que se calme y se tranquilice, y ahi ya vienen los castigos, le decimos que no puede; a ella le gusta dormir con la abuela entonces la castigamos que no puede dormir con la abuela, que no puede ver el celular, que no puede salir al parque, cosas asi (Andrés, pareja heterosexual).

\section{Subcategoría emergente: educación en sexualidad y diversidad sexual}

Finalmente, ambas parejas mencionaron la educación en sexualidad y diversidad sexual, manifestando que ésta es sólo cuestión de gustos. Sin embargo, se encontró que la pareja homosexual educaba a su hija en este tema, mientras que la pareja heterosexual aún no veía la necesidad de hablar sobre ello:

(...) es un gusto y nadie piensa igual que nadie, cada cabeza es un universo, cada cabeza es un mundo (Pepe, pareja homosexual).

(...) hasta el momento, no la hemos educado sobre sexualidad, ella hasta ahora no nos ha preguntado nada, ni nosotros hemos visto la necesidad de decirle, pues en algún momento, llegará el momento de hablarle pero ella todavía está muy pequeña para eso (Andrés, pareja heterosexual). 
Creencias de género, prácticas de crianza y apoyo social percibido: el caso de una pareja homosexual y una heterosexual

\section{Apoyo social percibido}

Al preguntar sobre el apoyo social percibido de tipo emocional, material e informacional en la crianza de sus hijas, ambas parejas coincidieron en que sus familias, y especialmente las abuelas de las niñas, habían sido los mayores referentes de todos los tipos de apoyo en la crianza. Además, expresaron percibir aceptación y apoyo por parte de la familia extensa, especialmente de aquellos que han vivido situaciones similares:

(...) vivimos con la abuela de Pepe y la mamá de Pepe (...). La mamá de Pepe es una persona indispensable en la crianza de la niña, nos ha ayudado mucho y fuera de ayudarnos como que ella trata de inculcarle a la niña la idea de que ella tiene dos papás (Rick, pareja homosexual).

\section{Categoría emergente: creencias conservadoras relacionadas con la religión}

Para terminar, es importante señalar que ambas parejas mencionaron que las creencias conservadoras relacionadas con la religión habían sido un obstáculo en la conformación de sus hogares:

(...) frente a la familia fue como el tema de la religión, porque mi familia por parte de mi mamá que es como la más cercana, eran súper católicos y la familia de mi esposo es cristiana, entonces como que ahi no querian y no querian, y me decian no: sepárense, sepárense" (Emilia, pareja heterosexual).

(...) la que me crío a mi fue mi abuela y mi abuela es una mujer muy religiosa (...) cuando tú sigues la religión, la religión tiene algo estipulado, que si no es asi no es de ninguna otra manera (...) (Pepe, pareja homosexual).

\section{Discusión}

Los resultados permitieron identificar las categorías deductivas previamente establecidas, comenzando por las creencias de género frente al rol de los hombres y mujeres en la sociedad, el contexto económico, el hogar y la crianza de los hijos, continuando por las prácticas de crianza usadas por los padres (comunicación, cuidado y educación) y finalizando con el apoyo social percibido de tipo emocional, informacional y material que les brindaban sus familias. 
[166] Juan Sebastian Rueda-Toro, María Isabel Arana, Nathalia Buitrago, María del Mar Sánchez, Ana María Pineda, Linda Teresa Orcasita

Además, se retoman las unidades de análisis emergentes: la subcategoría educación en sexualidad y diversidad sexual y la categoría creencias conservadoras relacionadas con la religión, las cuales fueron construidas de manera inductiva a partir de lo expresado por las parejas; también, se presentan las bondades y limitaciones del método y se plantean conclusiones y recomendaciones derivadas de la investigación

En un primer momento se resalta el hecho de que, si bien no con las mismas palabras, ambas parejas coincidieron en los temas presentes en sus respuestas: las narrativas de la pareja homosexual dieron cuenta, al igual que la heterosexual, de que mantenían creencias de género tradicionales frente a los roles que cumplen los hombres y las mujeres en distintos contextos. A su vez, las respuestas frente a las prácticas de crianza resultaron homogéneas, en cuanto a que la comunicación en la crianza fue percibida como abierta y la necesidad de establecer límites y enseñar el respeto como indispensables por ambas parejas. En relación con el apoyo social percibido, las parejas hicieron referencia a sus familias, especialmente a las abuelas, como los principales referentes de apoyo emocional, material e informacional; sin embargo, a partir de sus discursos emergió que las creencias religiosas conservadoras de algunos de sus familiares habían representado un obstáculo para sus procesos personales y de pareja. Finalmente, la única diferencia que se resalta en las repuestas de ambas parejas es que la pareja homosexual ya había iniciado la educación sexual de su hija, mientras que la pareja heterosexual aún no lo consideraba necesario.

Ahora bien, con el objetivo de profundizar en los resultados obtenidos en cada categoría, en cuanto a las creencias de género se encontró que el hombre era asociado, por ambas parejas, con características como autoridad, responsabilidad, capacidad de ser guia y carácter fuerte y ser estricto, mientras que la mujer fue relacionada con ser ejemplo, apoyo, sabiduría y comprensión y brindar afecto. Esto guarda relación con los hallazgos de Esquila et al. (2015) quienes encontraron que el hombre es considerado generalmente como proveedor de recursos del hogar y autoridad principal, otorgándole la responsabilidad en la toma de decisiones familiares, la generación de ingresos económicos y la capacidad de resolver asuntos del ámbito público, mientras que a la mujer 
se le atribuye lo subjetivo, intuitivo, pasivo, tierno, sensible, dócil, receptivo, empático, dependiente, emocional y conservador.

Los resultados difieren con las afirmaciones de Fonseca, Quintero y Zarza (como se citó en Esquila et al., 2015), quienes mencionan que actualmente los roles de género se han ido redefiniendo, dejando de ser tan claros en la sociedad actual y comenzando a ser subjetivos, ya que tanto en la pareja homosexual como en la heterosexual, las creencias de género con respecto al hombre y a la mujer guardan una gran similitud, además de mantenerse en la idea tradicional del hombre como el representante de fuerza y protección y la mujer del afecto y la comprensión, lo que concuerda con los hallazgos de Sánchez y Cárdenas (2017) frente a las representaciones sociales en estudiantes, asociadas al rechazo de formas de ser mujer distintas a las tradicionales.

En consecuencia, es posible afirmar que las dos parejas contaban con creencias hegemónicas y patriarcales relacionadas con el género, provenientes de la construcción social de lo que significa ser hombre y ser mujer hoy en la sociedad colombiana, lo que coincide con las afirmaciones de Acosta et al. (2017), Acevedo-Correa et al. (2018), Laguna (2018), Martínez (2018) y Castaño-Suárez et al. (2018) cuando concluyen que la orientación sexual no influye en que las parejas homosexuales puedan ejercer los mismos roles de género en la crianza de un hijo si se tiene en cuenta que dichos roles provienen, en gran medida, de las creencias anteriormente mencionadas.

Lo anterior pone de manifiesto lo arraigadas que se mantienen las ideas patriarcales de búsqueda de una "coherencia sexo-genérica" para los hombres y mujeres, en donde lo que los hombres piensan, dicen y llevan al acto tendría que ver con la fortaleza, autoridad y responsabilidad, mientras que en el caso de las mujeres tendría que ver con el afecto, la comprensión y la sabiduría. Estas creencias, de acuerdo con los resultados de este estudio, no cambian debido a la orientación sexual de dichas parejas, lo que pone de manifiesto la importancia de la educación y búsqueda de equidad de género para todas las parejas, inclusive las homosexuales, en las que las desigualdades de género pueden gestarse a partir de la interiorización y reproducción de roles de género rígidos por cada uno de los integrantes de la pareja, al considerar que se debe cumplir el "rol femenino" o el "rol masculino", lo que estaría desconociendo 
que el género se constituye como una construcción sociocultural que atraviesa a cada quien a partir del derecho a elegir, desde sus propias características masculinas, femeninas y no binarias, la manera en que se identifica y expresa ante la sociedad (Orcasita, Sevilla, Acevedo-Velasco, Montenegro, Tamayo y Rueda-Toro, 2019).

En relación con las prácticas de crianza y la comunicación dentro de la familia, ambas parejas se refirieron a la utilización del diálogo como instrumento esencial para comunicarse y establecer una relación más estrecha, con lo que se corrobora lo enunciado por Herrera (como se citó en Garcés y Palacio, 2010 y Smietana, 2010), quienes mencionan la instrumentalidad y utilidad del diálogo en la comunicación y en la expresión de afecto de las familias, y la importancia de la comunicación abierta para el bienestar de las relaciones entre padres, hijos e hijas. Frente al cuidado, ambas parejas manifestaron tener un acompañamiento afectuoso hacia sus hijas, pues las orientaban y educaban a través del afecto, lo que se veía reflejado en la percepción que tenían de sus prácticas de crianza como bonitas y afectivas.

Según Izzedin y Pachajoa (2009), las prácticas de crianza se relacionan con la normatividad y forma de educar que siguen los padres frente al comportamiento de los hijos, siendo estas portadoras de significaciones sociales. Así, se corroboró que el establecimiento de normas en las dos familias que participaron de la investigación se daba por medio de consensos logrados a través del diálogo entre ambos integrantes de la pareja, lo que se relaciona con el concepto de desalienación de los roles de crianza, pues ambas parejas organizaban sus tareas de acuerdo con sus realidades particulares y no a roles tradicionales (Laguna, 2018). No obstante, cabe mencionar que hubo una diferencia en el componente educativo, pues la educación en sexualidad y diversidad sexual difirió en ambas familias, ya que mientras que en la pareja heterosexual la sexualidad no se consideraba relevante en la educación de su hija debido a su edad, en la pareja homosexual se buscaba educar a la hija en el respeto hacia la diversidad desde una edad temprana. De esta forma, con respecto a la educación, se resalta que, si bien toda familia incluye en su dinámica normas que guían a sus hijos, cada una introduce los temas que cree pertinentes para formarlos y lo hace de acuerdo con sus necesidades y realidades particulares. 
Con respecto al apoyo social percibido, Orcasita y Uribe (2010) afirman que este tiene que ver con los actores (familia, amigos, instituciones) cercanos a la persona, los cuales puedan brindar apoyo emocional, de escucha, información, dinero, entre otros. Las parejas del estudio coincidieron en que los mayores referentes de apoyo emocional, consejo y guía en la crianza de sus hijos eran sus familias, especialmente las abuelas de las niñas, lo que resalta que la importancia del apoyo social familiar en el desarrollo de los hogares colombianos no discrimina por orientación sexual, siendo igualmente importante para una pareja homosexual como para una heterosexual contar con la ayuda brindada por su familia extensa. De esta manera, se recomienda que futuras investigaciones indaguen sobre el apoyo social familiar dirigido a la crianza de los hijos de personas gais y lesbianas; debido al estigma, los prejuicios y la discriminación, es posible que algunas familias no cuenten con el apoyo de sus parientes.

Los participantes concordaron en que los mayores obstáculos en la conformación de su familia tenían relación con creencias conservadoras de tipo religioso. Esto se asocia con los planteamientos de Zapata (2013) en cuanto a la necesidad de generar concepciones de familia menos restrictivas, dando apertura a la diversidad, especialmente teniendo en cuenta que en Colombia "los niños, las niñas y los jóvenes que crecen en familias homoparentales enfrentan una difícil situación” (p. 15) porque aún existe discriminación proveniente de creencias conservadoras hegemónicas y patriarcales en referencia a lo que deberían ser o no las familias, como es el caso de la pareja heterosexual que, a pesar de conformar una familia nuclear (padre, madre e hija), expresaron haber sentido discriminación por miembros de su familia extensa hacia ellos, por haber sido padres a una temprana edad.

Se resaltan aspectos del cuidado de los participantes que asumieron los investigadores en este estudio para favorecer un mayor acercamiento a la población de hombres gais, especialmente frente a la crianza de sus hijos e hijas, tema por el que llegaban a considerar su participación en el estudio como una potencial amenaza para sus familias, al poder llegar a generarles una mayor visibilización por parte de otros actores que podrían discriminarlos. Ante esto, se tuvo un primer acercamiento a las parejas y a sus contextos, no solo para 
prever posibles amenazas, sino para hacerlo de manera colaborativa con los participantes, disminuyendo sus niveles de ansiedad y promoviendo su participación voluntaria y anónima en el estudio. De igual modo, se reconoce la importancia del diseño empleado de estudio de caso fenomenológico (Stake, 2007) porque permitió la comprensión del fenómeno de estudio en dos casos específicos; sin dejar de lado la comparación entre estos, pero sin hacerla el objetivo central de la investigación.

En relación con las limitaciones del estudio, se reconoce el difícil acceso a la muestra, especialmente de la pareja homosexual, debido a las amenazas de sanción social por ser una familia que enfrenta constantemente el estigma social; implicó un contacto previo en el que se aseguró a los participantes que la información obtenida no sería divulgada públicamente con sus nombres reales. Se recomienda para futuras investigaciones ampliar la muestra teniendo en cuenta más parejas homosexuales, además de incluir parejas de mujeres que favorezcan la comprensión de estos fenómenos en familias cuyo subsistema parental está conformado únicamente por mujeres, teniendo en cuenta medidas preventivas y éticas para evitar las amenazas que para la población de personas homosexuales pueden derivarse de su participación en un estudio semejante.

$\mathrm{Al}$ analizar los roles de género en la crianza de los hijos de la pareja homosexual y la pareja heterosexual, es posible afirmar que las prácticas de crianza y las creencias de género enunciadas por ambas contienen un mayor número de similitudes (Herrera, Pavicevic y Sciaraffia, 2018), pues la única diferencia identificada radica en que la pareja homosexual ya brindaba educación sexual a su hija, mientras que la heterosexual no. Esto último se atribuye a la orientación sexual de los padres y la posible vivencia del estigma, los prejuicios y la discriminación que han presenciado por parte de la sociedad, por lo que buscaban que su hija tuviera conocimientos al respeto sustentados en los derechos que cobijan la diversidad humana.

Las similitudes encontradas en las creencias de género mostraron que ambas parejas relacionaron al hombre con roles de fuerza y a la mujer con roles de afecto, lo que podría decirse, repercute de la misma forma en las prácticas de crianza empleadas por ambas parejas: en la heterosexual las tareas de cuidado 
eran llevadas a cabo por la mujer y las de responsabilidad económica por el hombre, y en la pareja homosexual ocurría de la misma manera, ya que uno de los participantes aseguraba ser "la mujer de la relación" y el otro "el hombre". No obstante, cuando hablaban de la comunicación con sus hijas las dos parejas hicieron referencia al diálogo abierto y a la expresión de emociones, y cuando se expresaron frente a la educación se refirieron al establecimiento de normas. Estos y otros ejemplos ponen de manifiesto la existencia de pensamientos y formas de actuar tanto "femeninas" como "masculinas" en los participantes, lo que requerirá de una mayor profundización a partir de la investigación teórica y práctica, que se recomienda esté encaminada a develar las consecuencias de las creencias de género en las prácticas de cuidado de parejas jóvenes y los significados que estos otorgan a los roles que cumplen como padres y madres independientemente de su orientación sexual.

Se identificó la importancia del apoyo social familiar para ambas parejas, especialmente en la consolidación y desarrollo de sus hogares, al constituirse como fuente de apoyo emocional, material e informacional frente a las tensiones propias de las dinámicas internas y externas al núcleo familiar, y se reconocieron las creencias conservadoras relacionadas con la religión como posibles obstáculos ante la constitución y desarrollo de hogares con padres jóvenes de manera independiente a la orientación sexual de estos.

Se recomienda para futuras investigaciones procurar medidas éticas que promuevan la participación de personas homosexuales mientras se sienten seguros para hacerlo, como la utilización de espacios de encuentro privados y escogidos y/o aprobados previamente por los participantes. De igual modo, se considera pertinente continuar con un análisis a profundidad de cómo afectan a largo plazo las creencias de género de padres gais y madres lesbianas, las prácticas de crianza que emplean con sus hijos e hijas y, de ser posible, ofrecer orientación a las parejas participantes en conceptos como diversidad sexo-genérica, que les permita comprender la importancia de reconocer el género como dinámico y flexible en la construcción y expresión de la identidad de todos los seres humanos.

En relación con esto, la recomendación de formar a las parejas para favorecer su reconocimiento del género como dinámico y flexible, se extiende para 
todos los psicólogos y profesionales que trabajan con parejas, independientemente de su orientación sexual, por el mejoramiento de su salud mental, bienestar y la visibilización de aquellas poblaciones excluidas por prácticas no aceptadas o no avaladas por los discursos dominantes. La educación en género, sexualidad y diversidad sexo-genérica dirigida a trabajar las creencias de género, podría constituirse como una herramienta que provea mayor bienestar a la hora de asumir el rol de padres y madres mediante la comprensión de que estos no implican necesariamente contar con características rígidas de lo "femenino" y/o "masculino", sino que pueden ser construidos por cada uno de acuerdo a sus formas particulares de ser. Esto, a su vez, podría favorecer una crianza más inclusiva y que reconozca la importancia de la educación sexual y el respeto por la diversidad humana.

Finalmente, esta investigación buscó establecer un acercamiento al estudio de la influencia de las creencias de género y el apoyo social percibido en las prácticas de crianza de familias que no se encuentran dentro de los parámetros sociales hegemónicos considerados "tradicionales" (papá, mamá e hijos y familias planeadas), lo que puede sentar una base para la generación de nuevos estudios que estén dirigidos a resolver necesidades prácticas de dichas familias que cada día constituyen una mayor proporción de la sociedad colombiana. Además, los resultados de la investigación reflejan la importancia de continuar realizando evaluaciones empíricas que permitan reconocer los matices y variaciones en las creencias de diversos tipos de parejas.

\section{Referencias}

Acevedo-Correa, L., Marín-Castillo, J., Heredia-Quintana, D., Gómez-Vargas, M., Múnera-Rúa, N., Correa-Sierra, L. y Medina, J. (2018). La adopción homoparental en Colombia: presupuestos jurídicos y análisis de la idoneidad mental. Anuario de Psicología Jurídica, 28(1), 58-65. doi: https://doi.org/10.5093/ apj2018a8. Recuperado de https://journals.copmadrid.org/apj/art/apj2018a8

Acosta, M., Costales, Z. y Rosales, B. (2017). Familia Homoparental en Cuba: Una realidad no contada. Revista latinoamericana de comunicación, 135, 89104. Recuperado de https://revistachasqui.org/index.php/chasqui/article/ view/3184/2934 
Creencias de género, prácticas de crianza y apoyo social percibido: el caso de una pareja homosexual y una heterosexual

American Psychological Association (2017). Las personas trans y la identidad de género. Washington, EU.: American Psychological Association. Recuperado de: http:// www.apa.org/topics/lgbt/transgenero.aspx

Andrade, Á. M. y Uribe, M. A. (2015). Las familias homoparentales y el cuidado. Prospectiva, 20, 351-374. Doi: http://doi.org/10.25100/prts.v0i20.946. Recuperado de https://dialnet.unirioja.es/servlet/articulo?codigo $=5876990$

Astaíza-Martínez, A. F. (2016). Situación actual de los tratamientos psicológicos para la homosexualidad. Revista de Psicología Universidad de Antioquia, 8(2), 173194. doi: 10.17533/udea.rpsua.v8n2a10. Recuperado de https://aprendeenlinea.udea.edu.co/revistas/index.php/psicologia/article/view/327891

Bonilla, E. y Rodríguez, P. (2013). Más allá del dilema de los métodos: la investigación en ciencias sociales. Bogotá, Colombia: Ediciones Uniandes. Recuperado de https:/www.scribd.com/document/375854141/BONILLA-RODRIGUEZMas-Alla-Del-Dilema-de-Los-Metodos-UPA

Castaño-Suárez, M., Sánchez-Trujillo, M. P. y Viveros-Chavarría, E. F. (2018). Familia homoparental, dinámicas familiares y prácticas parentales. Revista Latinoamericana de Estudios de Familia, 10(2), 51-70. doi: 10.17151/rlef.2018.10.2.5. Recuperado de http://revlatinofamilia.ucaldas.edu.co/downloads/Rlef10(2)_4. pdf

Ceballos, M. (2012). Ser madres y padres en familias homoparentales: análisis del discurso de sus percepciones sobre la educación de sus hijos e hijas. Ensayos, 27, 143-158. Recuperado de http://digibuo.uniovi.es/dspace/bitstream/10651/18637/1/246-1076-1-PB.pdf

Colegio Colombiano de Psicólogos (2014). Efectos de la adopción homoparental sobre el desarrollo integral del niño, niña o adolescente en Colombia. Recuperado de https://issuu.com/colpsic/docs/respuesta_a_icbf2_vs._dc_-_20-10$20 / 1$ ? $=18058890 / 33373162$

Colegio Colombiano de Psicólogos (2009). Deontología y Bioética del ejercicio de la Psicología en Colombia. Recuperado de http://www.infopsicologica.com/documentos/2009/Deontologia_libro.pdf

Congreso de la República de Colombia (2006). Ley 1090 del 6 de septiembre de 2006 por la cual se reglamenta el ejercicio profesional psicológico, se dicta el código deontológico y bioético. Bogotá, Colombia.: Congreso de la República de Colombia. Recuperado de http://colpsic.org.co/aym_image/files/LEY_1090_DE_2006.pdf

Córdoba, H. (2019). Hacia un breve glosario Queer: algunas nociones acerca del género, la sexualidad y la teoría queer. Análisis, 52(96), 95-121. 
[174] Juan Sebastian Rueda-Toro, María Isabel Arana, Nathalia Buitrago, María del Mar Sánchez, Ana María Pineda, Linda Teresa Orcasita

Esquila, A., Zarza, S., Villafaña, G. y Oudhof, H. (2015). La identidad y rol de género en la relación de pareja: un estudio generacional sobre la permanencia en el matrimonio. Revista Electrónica de Psicología Iztacala, 18(4), 15071538. Recuperado de http://www.revistas.unam.mx/index.php/repi/article/ view/53442/47533

Garcés, M. y Palacio, J. (2010). La comunicación familiar en asentamientos subnormales de montería (Colombia). Psicología desde el Caribe, 25, 1-29. Recuperado de http://www.redalyc.org/articulo.oa?id=21315106002

García, C. I. (2007). Diversidad sexual en la escuela. Dinámicas pedagógicas para enfrentar la homofobia / Colombia. Bogotá, Colombia: Colombia Diversa.

Gómez, A. (2004). Diversidad familiar y homoparentalidad. Revista Pediatría de Atención Primaria, 6(23), 361-365. Recuperado de http://archivos.pap.es/files/1116-368-pdf/381.pdf

González, T., Posada, P., Rojas, V., Rueda, J. S. y Soto, A. M. (2017). Dimensiones de los estereotipos de género: comportamiento sexual; competencias o capacidades en estudiantes universitarios de la Pontificia Universidad Javeriana Cali (Proyecto Investigación en Psicología I). Pontificia Universidad Javeriana, Cali, Colombia.

Herrera, M. C., Pavicevic, Y. y Sciaraffia, V. (2018). "Soy un papá súper normal”: Experiencias parentales de hombres gay en Chile. Polis (Santiago), 17(50), 111-137. Doi: https://dx.doi.org/10.4067/S0718-65682018000200111. Recuperado de https://scielo.conicyt.cl/scielo.php?script=sci_arttext\&pi$\mathrm{d}=$ S0718-65682018000200111\&lang $=\mathrm{pt}$

Hernández, R., Fernández, C. y Baptista, M. (2010). Metodología de la investigación. México D. F., México: McGrawGill. Recuperado de https://www.esup.edu. pe/descargas/dep_investigacion/Metodologia\%20de\%20la\%20investigaci\%C3\%B3n\%205ta\%20Edici\%C3\%B3n.pdf

Instituto Colombiano de Bienestar Familar (2006). El Arte de Criar Hijos con amor. Recuperado de https://www.icbf.gov.co/sites/default/files/pautasdecrianza001.pdf

Izzedin, R. y Pachajoa, A. (2009). Pautas, prácticas y creencias acerca de crianza... Ayer y hoy. Revista de Psicología, 15(2), 109-115. Recuperado de http://pepsic. bvsalud.org/scielo.php?script=sci_arttext\&pid=S1729-48272009000200005

Jelin, E. (2010). Pan y afectos: la transformación de las familias. Buenos Aires, Argentina: Fondo de Cultura Económica.

Laguna, O. (2018). Paternidad de hombres gay: ¿Los albores de una neoparentalidad?. Polis (Santiago), 17(50), 139-160. Doi: https://dx.doi.org/10.4067/ 
Creencias de género, prácticas de crianza y apoyo social percibido: el caso de una pareja homosexual y una heterosexual

S0718-65682018000200139. Recuperado de https://scielo.conicyt.cl/scielo. php?script=sci_arttext $\&$ pid=S0718-65682018000200139\&lang=pt

Martínez, J. (2018). Efectos de adopción y crianza homoparental (Tesis de especialización). Universidad Nacional de Colombia, Bogotá, Colombia. Recuperado de http://bdigital.unal.edu.co/62128/7/1053778728.2018.pdf

Martínez-Salgado, C. (2012). El muestreo en investigación cualitativa. Principios básicos y algunas controversias. Ciência \& Saúde Coletiva, 17(3), 613-619. Recuperado de http://www.scielo.br/pdf/csc/v17n3/v17n3a06.pdf

Mieles, M., Tonon, G. y Alvarado, S. (2012). Investigación cualitativa: el análisis temático para el tratamiento de la información desde el enfoque de la fenomenología social. Universitas Humanistica, 74, 195-225. Recuperado de http:// www.scielo.org.co/pdf/unih/n74/n74a10.pdf

Ministerio de Salud. (1993). Resolución No 008439 de 1993. Recuperado de http:// www.unisabana.edu.co/fileadmin/Documentos/Investigacion/comite_de_etica/Res_8430_1993_-_Salud.pdf

Observatorio de Políticas de las Familias y Departamento Nacional de Planeación (2015). Tipologias de Familias en Colombia: Evolución 1993-2014. Recuperado de https://observatoriodefamilia.dnp.gov.co/Documents/Documentos\%20 de\%20trabajo/D3-tipologias-evolucion_dic3-(1).pdf

Orcasita, L. T., Sevilla, T. M., Acevedo-Velasco, V. E., Montenegro, J. L., Tamayo, M. C. y Rueda-Toro, J. S. (2019). Apoyo social familiar para el bienestar de hijos gays e hijas lesbianas. Revista Latinoamericana de Ciencias Sociales, Niñez y Juventud, 18(2), 1- 23. Doi: http://dx.doi.org/10.11600/1692715x.18205. Recuperado de http://revistaumanizales.cinde.org.co/rlcsnj/index.php/Revista-Latinoamericana/article/view/3966

Orcasita, L. y Uribe, A. (2010). La importancia del apoyo social en el bienestar de los adolescentes. Psychologia. Avances de la disciplina, 4(2), 69-82. Recuperado de http://www.redalyc.org/pdf/2972/297224090010.pdf

Ríos, M. (2017). Y, ¡cómo lo hacen? Familias homoparentales con niños en Lima: constitución y estrategias de crianza y socialización. Estudio de caso de familias de clase media y media alta en distritos de Lima Metropolitana y El Callao (Tesis de maestría). Pontificia Universidad Católica del Perú, Lima, Perú. Recuperado de http://hdl.handle.net/20.500.12404/8771

Sánchez, R. y Cárdenas, E.K. (2017). Representaciones sociales de mujer en estudiantes de quinto grado de una institución educativa de Tunja. Revista de Psicología Universidad de Antioquia, 9(2), págs. 67-86. doi: 10.17533/udea.rp.v9n2a05. 
[176] Juan Sebastian Rueda-Toro, María Isabel Arana, Nathalia Buitrago, María del Mar Sánchez, Ana María Pineda, Linda Teresa Orcasita

Recuperado de http://aprendeenlinea.udea.edu.co/revistas/index.php/psicologia/article/view/329752

Smietana M. (2010). Estrategias de comunicación de familias con padres gays/madres lesbianas (Communication strategies of families with gay fathers/lesbian mothers), X Congreso Español de Sociología, Proceedings: Pamplona (Spain).

Stake, R. E. (2007). Investigación con estudio de casos. Madrid, Espańa: Ediciones Morata.

Zapata, B. (2013). Las voces de la homoparentalidad. Resultados preliminares de una investigación. Revista Trabajo Social, 15, 41-58. Recuperado de https://revistas. unal.edu.co/index.php/tsocial/article/view/42570/44097 